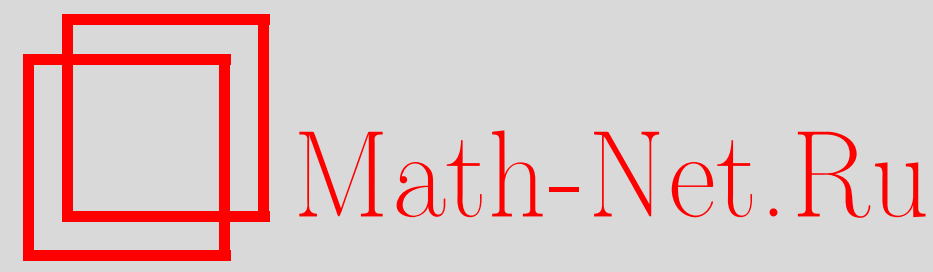

В. А. Добрынский, Существование двумерных топологически перемешивающих аттракторов у некоторых кусочно линейных отображений плоскости, Изв. РАН. Сер. матем., 1998, том 62, выпуск 6, 53-58

DOI: https://doi.org/10.4213/im220

Использование Общероссийского математического портала Math-Net.Ru подразумевает, что вы прочитали и согласны с пользовательским соглашением

http://www . mathnet.ru/rus/agreement

Параметры загрузки:

IP : 18.208 .226 .222

26 апреля 2023 г., 04:24:53 
УДК 517.9

\author{
В. А. Добрынский
}

\title{
Существование двумерных топологически перемешивающих аттракторов у некоторых кусочно линейных отображений плоскости
}

\begin{abstract}
Доказано существование двумерных топологически перемешивающих аттракторов у эндоморфизмов плоскости, образованных путем сцепления двух одинаковых одномерных унимодальных кусочно линейных отображений.

Библиографиия: 4 наименования.
\end{abstract}

Одним из наиболее изученных классов одномерных систем является семейство кусочно линейных отображений вида $\tau \rightarrow 1-a|\tau|$. Это связано с тем, что, обладая по сути дела столь же сложной динамикой, как и гладкие отображения, кусочно линейные намного проще и легче поддаются изучению аналитическими методами. Сказанное в еще большей мере справедливо для многомерных динамических систем. А учитывая, как мало известно о свойствах даже двумерных кусочно линейных отображений, вполне понятен тот интерес, который они вызывают.

В последнее время в литературе широко обсуждаются эффекты, возникаюшие при сцеплении ряда идентичных одномерных отображений.

Пусть $f_{a}: \tau \rightarrow f_{a}(\tau)$ - однопараметрическое семейство отображений прямой $\mathbb{R}^{1}$ в себя. Сцепление пары таких отображений может быть произведено различными способами. Чаше всего рассматриваются отображения вида

$$
(x, y) \rightarrow\left(f_{a}(x)+\zeta\left(f_{a}(y)-f_{a}(x)\right), f_{a}(y)+\zeta\left(f_{a}(x)-f_{a}(y)\right)\right),
$$

где $(x, y) \in \mathbb{R}^{2}[1],[2]$. Очевидно, они обладают свойством симметрии (относительно замен $x \rightarrow y, y \rightarrow x)$, вследствие чего при любых значениях параметров $a$ и $\zeta$ имеют одномерное инвариантное многообразиев виде главной диагонали $x=y$, на которой они совпадают с $f_{a}$. Ниже исследуются отображения $(1)$ с $f_{a}(\tau)=1-a|\tau|$, т.е. рассматривается двупараметрическое семейство кусочно линейных эндоморфизмов плоскости вида

$$
\Phi:(x, y) \rightarrow(1-a(1-\zeta)|x|-a \zeta|y|, 1-a(1-\zeta)|y|-a \zeta|x|),
$$

где $a$ и $\zeta$ - параметры такие, что $0<a<2$ и $0<\zeta<1$. Толчком для изучения свойств этого класса отображений послужили статьи [1], [2], в которых представлен ряд результатов численного анализа свойств двух семейств кусочно линейных отображений (из которых семейство, рассматриваемое в [1], такое же, как (2), а рассматриваемое в [2] совпадает с $\Phi$ после соответствующей линейной замены координат). В частности, в обеих работах выдвинуто предположение о сушествовании значений параметров таких, что соответствуюшие им отображения имеют

(C) В.А. Доврынский 1998 
двумерные хаотические аттракторы. Кроме того, в [1] сформулирована дополнительно гипотеза о топологической транзитивности аттракторов и плотности периодических точек на них.

Основным результатом является следующая теорема.

ТеоремА. Для произвольного $a \in\left(\frac{1+\sqrt{5}}{2}, 2\right)$ существует $\zeta_{a} \in\left(0, \frac{a-1}{2 a}\right)$ maкое, что для любых $\zeta \in\left(0, \zeta_{a}\right)$ отображение $\Phi$ имеет хаотический странный аттрактор $\Lambda$, представляющий собой четырехугольник (ромбоид, стороны которого образованы парами отрезков двух первых образов критического множества отображения $\Phi)$, на котором $\Phi$ является топологически перемешивающим отображением.

Другими словами, в пространстве параметров есть такая область, что для каждого набора параметров из этой области для отображения $\Phi$ в фазовом пространстве существует область $U$ такая, что $\Phi(\mathrm{Cl} U) \subset \operatorname{Int} U, \bigcap_{j=0}^{\infty} \Phi^{j}(U)=\Lambda$ и для любого конечного покрытия $\Lambda$ окрестностями $V_{i}, i=\overline{1, N}$, открытыми в $\Lambda$, найдется натуральное $M$ такое, что $\Phi^{p}\left(V_{i}\right) \cap V_{j} \neq \varnothing$ для произвольных $i, j \in\{1, \ldots, N\}$ и $p \geqslant M$. Здесь и далее $\mathrm{Cl} U$ и $\operatorname{Int} U$ обозначают замыкание и внутренность множества $U$ соответственно.

СлЕДСТВИЕ. Если $a \in\left(\frac{1+\sqrt{5}}{2}, 2\right)$ u $\zeta \in\left(0, \zeta_{a}\right)$, mо $\Lambda$ - топологически транзитивное множество, на котором плотны периодические точки.

В дальнейшем мы ограничимся изучением $\Phi$, соответствуюших значениям $a$ и $\zeta$ из интервалов $\left(\frac{1+\sqrt{5}}{2}, 2\right)$ и $\left(0, \frac{a-1}{2 a}\right)$. При таких $a$ и $\zeta$ оба собственных числа дифференщиала $D \Phi$ (там, где он сушествует) по модулю больше 1 , а отображение $\Phi$ имеет пару неподвижных точек

$$
\left(\frac{1}{1+a}, \frac{1}{1+a}\right), \quad\left(\frac{1}{1-a}, \frac{1}{1-a}\right)
$$

и пару периодических периода 2 точек

$$
\left(\frac{1+a(1-2 \zeta)}{1+a a(1-2 \zeta)}, \frac{1-a(1-2 \zeta)}{1+a a(1-2 \zeta)}\right), \quad\left(\frac{1-a(1-2 \zeta)}{1+a a(1-2 \zeta)}, \frac{1+a(1-2 \zeta)}{1+a a(1-2 \zeta)}\right) .
$$

Обозначим $K_{\Phi}=K_{x} \cup K_{y}$, где $K_{x}=\{(x, y): x=0\}, \quad K_{y}=\{(x, y)$ : $y=0\}$, критическое множество отображения $\Phi$. (Напомним, что критическим для отображения $\Psi$ называется множество точек, в которых якобиан $D \Psi$ обращается в нуль или же вообще не существует [3], [4].) Поскольку $\Phi:(t, 0) \rightarrow$ $(1-a(1-\zeta)|t|, 1-a \zeta|t|)$ и $\Phi:(0, t) \rightarrow(1-a \zeta|t|, 1-a(1-\zeta)|t|)$, то

$$
\begin{aligned}
& \Phi(\{(x, y): y=0\})=\left\{(x, y): y=1+\frac{\zeta}{1-\zeta}(x-1), x \leqslant 1\right\} \\
& \Phi(\{(x, y): x=0\})=\left\{(x, y): y=1+\frac{1-\zeta}{\zeta}(x-1), x \leqslant 1\right\} .
\end{aligned}
$$

Объединение этих множеств дает $\Phi\left(K_{\Phi}\right)$. Нетрудно показать, что

$$
\Phi\left(\mathbb{R}^{2}\right)=\left\{(x, y): 1+\frac{1-\zeta}{\zeta}(x-1) \leqslant y \leqslant 1+\frac{\zeta}{1-\zeta}(x-1), x \leqslant 1\right\} .
$$


Обозначим $O=(0,0)$ начало координат и рассмотрим ромб $B D E F$, где $B=$ $(1,1), D=(1-2 \zeta,-1+2 \zeta), E=(-1,-1), F=(-1+2 \zeta, 1-2 \zeta)$. Оси координат делят ромб на четыре части. Обозначим $A=\left(0,1-\frac{\zeta}{1-\zeta}\right), C=\left(1-\frac{\zeta}{1-\zeta}, 0\right)$, $H=\left(0,-1+\frac{\zeta}{1-\zeta}\right), G=\left(-1+\frac{\zeta}{1-\zeta}, 0\right)$ точки пересечения осей координат со сторонами ромба и рассмотрим образы четырехугольников $O A B C, O C D H, O H E G$ и $A O G F$. Так как $\Phi$ - диффеоморфизм каждого из четырехугольников на его образ, достаточно найти координаты образов их вершин. Находим:

$$
\begin{gathered}
\Phi(0)=B=(1,1), \quad \Phi(A)=\Phi(H)=\left(1-a \zeta+\frac{a \zeta^{2}}{1-\zeta}, 1-a+2 a \zeta\right), \\
\Phi(B)=\Phi(E)=(1-a, 1-a), \quad \Phi(C)=\Phi(G)=\left(1-a+2 a \zeta, 1-a \zeta+\frac{a \zeta^{2}}{1-\zeta}\right), \\
\Phi(D)=\Phi(F)=(1-a(1-2 \zeta), 1-a(1-2 \zeta)) .
\end{gathered}
$$

Таким образом, $\Phi(O A B C)=\Phi(O H E G) \supset \Phi(O C D H)=\Phi(A O G F)$. Как следствие, получается, что $\Phi(B D E F)=\Phi(O A B C)$. При этом поскольку $A, C \in K_{\Phi}$, то $\Phi(A), \Phi(C) \in \Phi\left(K_{\Phi}\right)$. А так как $-1+2 \zeta<1-a+2 a \zeta<1$ при $1<a<2$, $0<\zeta<\frac{a-1}{2 a}$, то $\Phi(A) \in B D$, а $\Phi(C) \in B F$. Следовательно, $\Phi(O A B C) \subset B D E F$. Причем при достаточно малых $\zeta$ точки $\Phi(A)$ и $\Phi(C)$ находятся в малых окрестностях точек $D$ и $F$ соответственно.

Обратим внимание на то, что ромбоид $\Phi(O A B C)$ образован парами отрезков, принадлежаших $\Phi\left(K_{\Phi}\right)$ и $\Phi^{2}\left(K_{\Phi}\right)$. Ниже мы покажем, что $\Phi(O A B C)$ и есть то самое множество $\Lambda$, о котором упоминается в формулировке теоремы.

ЛЕмма 1. Для произвольных $a \in(1,2)$ u $\zeta \in\left(0, \frac{a-1}{2 a}\right)$ существует область $U \subset \mathbb{R}^{2}$ такая, что $U \supset B D E F, \Phi(\mathrm{Cl} U) \subset \operatorname{Int} U u \bigcap_{j=0}^{\infty} \Phi^{j}(\mathrm{Cl} U) \subset B D E F$.

ДокАЗАТЕЛЬСТво. Выбирая $U$ в виде $\gamma$-окрестности ромба $B D E F$, где $\gamma=$ $\gamma(a, \zeta)>0$ - достаточно малая величина, нетрудно убедиться в том, что $\Phi(\mathrm{Cl} U) \subset$ $\operatorname{Int} U$. Это означает также, что $\Phi^{j}(\mathrm{Cl} U) \subset \Phi^{j-1}(\mathrm{Cl} U)$ для любого натурального $j$. Более того, вычисление нескольких последовательных итераций $\mathrm{Cl} U$ показывает, что существует натуральное $N$ такое, что $\Phi^{N}(\mathrm{Cl} U) \subset B D E F$.

Рассмотрим отображения, обратные к $\Phi$. Имеется четыре таких отображения. Именно:

$$
\begin{array}{ll}
\Phi_{(1)}^{-1}:(x, y) \rightarrow(g(x, y), h(x, y)), & \Phi_{(2)}^{-1}:(x, y) \rightarrow(g(x, y),-h(x, y)), \\
\Phi_{(3)}^{-1}:(x, y) \rightarrow(-g(x, y),-h(x, y)), & \Phi_{(4)}^{-1}:(x, y) \rightarrow(-g(x, y), h(x, y)),
\end{array}
$$

где

$$
g(x, y)=\frac{1}{a}-\frac{(1-\zeta) x}{a(1-2 \zeta)}+\frac{\zeta y}{a(1-2 \zeta)}, \quad h(x, y)=\frac{1}{a}-\frac{(1-\zeta) y}{a(1-2 \zeta)}+\frac{\zeta x}{a(1-2 \zeta)} .
$$

Все это линейные сжатия. Определены они на $\Phi\left(\mathbb{R}^{2}\right)$ и отображают его на разные квадранты $\mathbb{R}^{2}$. Точнее,

$$
\begin{aligned}
& \Phi_{(1)}^{-1}\left(\Phi\left(\mathbb{R}^{2}\right)\right)=Q_{1}, \quad \Phi_{(2)}^{-1}\left(\Phi\left(\mathbb{R}^{2}\right)\right)=Q_{2} \\
& \Phi_{(3)}^{-1}\left(\Phi\left(\mathbb{R}^{2}\right)\right)=Q_{3}, \quad \Phi_{(4)}^{-1}\left(\Phi\left(\mathbb{R}^{2}\right)\right)=Q_{4}
\end{aligned}
$$


где

$$
\begin{array}{ll}
Q_{1}:=\{(x, y): x \geqslant 0, y \geqslant 0\}, & Q_{2}:=\{(x, y): x \geqslant 0, y \leqslant 0\}, \\
Q_{3}:=\{(x, y): x \leqslant 0, y \leqslant 0\}, & Q_{4}:=\{(x, y): x \leqslant 0, y \geqslant 0\} .
\end{array}
$$

Очевидно также, что $\Phi_{(1)}^{-1}(\Phi(O A B C))=O A B C, \Phi_{(2)}^{-1}(\Phi(O C D H))=O C D H$, $\Phi_{(3)}^{-1}(\Phi(O A B C))=O H E G$ и $\Phi_{(4)}^{-1}(\Phi(O C D H))=A O G F$.

Введем обозначение $\widetilde{K}_{\Phi}=K_{\Phi} \cap \Phi(B D E F)$. Действие $\Phi_{(j)}^{-1}, j=\overline{1,4}$, преобразует разбиение ромбоида $\Phi(B D E F)$ на части множеством $\widetilde{K}_{\Phi}$ в разбиения на части четырехугольников $O A B C, O C D H, O H E G, A O G F$ множествами $\Phi_{(j)}^{-1}\left(\widetilde{K}_{\Phi}\right), j=$ $\overline{1,4},-$ прообразами множества $\widetilde{K}_{\Phi}$. (Заметим кстати, что $\bigcup_{i=1}^{4} \Phi_{(i)}^{-1}\left(\widetilde{K}_{\Phi}\right)$ - не что иное, как подмножество множества точек $(x, y) \in \mathbb{R}^{2}$, координаты которых удовлетворяют одному из соотношений $1-a(1-\zeta)|x|-a \zeta|y|=0$ или $1-a(1-\zeta)|y|-a \zeta|x|=$ 0$.$) В результате получается новое разбиение ромбоида \Phi(B D E F)$ множествами $\widetilde{K}_{\Phi}, \bigcup_{i=1}^{4} \Phi_{(i)}^{-1}\left(\widetilde{K}_{\Phi}\right)$. Повторяя указанную операцию еще раз, мы построим разбиение ромбоида $\Phi(B D E F)$ множествами $\widetilde{K}_{\Phi}, \bigcup_{i=1}^{4} \Phi_{(i)}^{-1}\left(\widetilde{K}_{\Phi}\right)$ и $\bigcup_{j=1}^{4} \bigcup_{i=1}^{4} \Phi_{(j)}^{-1}$ $\left(\Phi_{(i)}^{-1}\left(\widetilde{K}_{\Phi}\right)\right)$, и т.д. Согласно описанной вьше процедуре построенное на $m$-м шаге разбиение является измельчением разбиения, построенного на $(m-1)$-м шаге. При этом множествами-делителями всякий раз являются подмножества $m$-х прообразов критического множества. Разбиения, получаемые таким образом, являются согласованными в том смысле, что под действием $\Phi$ границы их элементов переходят друг в друга, а сами элементы разбиения $m$-го порядка действием $\Phi_{(i)}^{-1}$ преобразуются в один или несколько (до четырех) элементов разбиения $(m+1)$-го порядка. Обозначим $\left\{\xi_{m}\right\}$ разбиение $m$-го порядка, a $\xi_{m}, \operatorname{diam}\left(\xi_{m}\right)$ и $\operatorname{mes}\left(\xi_{m}\right)-$ произвольный элемент разбиения, его диаметр и площадь.

Лемма 2. Для произвольного $a \in\left(\frac{1+\sqrt{5}}{2}, 2\right)$ существует $\zeta_{a} \in\left(0, \frac{a-1}{2 a}\right)$ такое, что для любих $\zeta \in\left(0, \zeta_{a}\right)$ соотношение $\left(\bigcup_{j=1}^{\infty} \Phi^{-j}((0,0))\right) \cap \xi_{m} \neq \varnothing$, где $\bigcup_{j=1}^{\infty} \Phi^{-j}((0,0))$ - множество всех прообразов точки $(0,0)$, выполняется для любого натурального $\mathrm{m}$.

ДокАЗАТЕЛЬСтво. Пусть $\xi_{m}-$ произвольный элемент разбиения $\left\{\xi_{m}\right\}$. В каждом из квадрантов ромба $B D E F$ отображение $\Phi$ растягивает элементы $\left\{\xi_{m}\right\}$ линейно по обоим направлениям. Поэтому, учитывая, что модули собственных чисел $D \Phi$ в $O A B C$ и $O H E G$ равны соответственно $а$ и $a(1-2 \zeta)$, а в $O C D H$ и $A O G F$ равны $a \sqrt{1-2 \zeta}$, находим, что под действием $\Phi$ площади увеличиваются в $a^{2}(1-2 \zeta)$ раз, т.е. $\operatorname{mes}\left(\Phi\left(\xi_{m}\right)\right)=a^{2}(1-2 \zeta) \operatorname{mes}\left(\xi_{m}\right)$. Так как при $\zeta=0$ $a^{2}(1-2 \zeta)=a^{2}>\left(\frac{1+\sqrt{5}}{2}\right)^{2}>2,6$ для $\frac{1+\sqrt{5}}{2}<a<2$, то для каждого $a \in\left(\frac{1+\sqrt{5}}{2}, 2\right)$ существует $\zeta_{a} \in\left(0, \frac{a-1}{2 a}\right)$ такое, что $a^{2}(1-2 \zeta)>2,2$ для всех $\zeta \in\left(0, \zeta_{a}\right)$. Следовательно, $\operatorname{mes}\left(\Phi\left(\xi_{m}\right)\right)>2,2 \operatorname{mes}\left(\xi_{m}\right)$.

Рассмотрим $\Phi\left(\xi_{m}\right)$. Возможны следуюшие три случая:

a) $\operatorname{Int} \Phi\left(\xi_{m}\right) \cap K_{\Phi}=\varnothing$;

б) Int $\Phi\left(\xi_{m}\right) \cap K_{\Phi} \neq \varnothing$, но либо Int $\Phi\left(\xi_{m}\right) \cap K_{x}=\varnothing$, либо же $\operatorname{Int} \Phi\left(\xi_{m}\right) \cap K_{y}=\varnothing$; 
в) $\operatorname{Int} \Phi\left(\xi_{m}\right) \cap K_{x} \neq \varnothing$ и $\operatorname{Int} \Phi\left(\xi_{m}\right) \cap K_{y} \neq \varnothing$.

Если имеет место а), то $\Phi\left(\xi_{m}\right)$ лежит в одном из квадрантов $B D E F$ и, следовательно, $\operatorname{mes}\left(\Phi^{2}\left(\xi_{m}\right)\right)>2,2 \operatorname{mes}\left(\Phi\left(\xi_{m}\right)\right)$. Предположим теперь, что справедливо б). Для определенности пусть $\operatorname{Int} \Phi\left(\xi_{m}\right) \cap K_{y}=\varnothing$. Тогда $K_{\Phi}$ делит $\Phi\left(\xi_{m}\right)$ на две части. Выбирая из них по площади большую (любую, если они равны), видим, что $\operatorname{mes}\left(\Phi^{2}\left(\xi_{m}\right)\right)>1,1 \operatorname{mes}\left(\Phi\left(\xi_{m}\right)\right)$. Таким образом, получается, что если $\Phi^{j}\left(\xi_{m}\right)$ таковы, что для каждого $j=1,2,3, \ldots$ выполняется а) или б), то $\operatorname{mes}\left(\Phi^{j}\left(\xi_{m}\right)\right)>1,1^{j} \operatorname{mes}\left(\xi_{m}\right)$, и, значит, $\operatorname{mes}\left(\Phi^{j}\left(\xi_{m}\right)\right)$ неограниченно растет. Последнее невозможно, следовательно, сушествует натуральное $J$ такое, что справедливо предположение в). Но в таком случае очевидно, что либо $\operatorname{Int} \Phi^{J}\left(\xi_{m}\right) \cap\{(x, y): y=x\} \neq \varnothing$, либо $\operatorname{Int} \Phi^{J}\left(\xi_{m}\right) \cap\{(x, y): y=-x\} \neq \varnothing$. Так как $\Phi(\{(x, y): y=-x\}) \subset\{(x, y): y=x\}$, последнее означает, что $L \stackrel{\text { df }}{=}$ $\operatorname{Int} \Phi^{J+1}\left(\xi_{m}\right) \cap\{(x, y): y=x\} \neq \varnothing$. Тогда, повторяя рассуждения, излагаемые в доказательстве леммы 3 , нетрудно убедиться в том, что существует натуральное $N$ такое, что $\Phi^{N}(L) \supset B D$, и, значит, $\Phi^{J+N+1}\left(\xi_{m}\right) \ni(0,0)$.

ЛЕмма 3. Для любых $a \in\left(\frac{1+\sqrt{5}}{2}, 2\right)$ u $\zeta \in\left(0, \frac{a-1}{2 a}\right)$ разбиения $\left\{\xi_{m}\right\}$ ромбоида $\Phi(O A B C)$ на части таковы, что для каждого натурального $m$ найдется другое натуральное число $M=M(m)$ такое, что для любого $\xi_{m} \in\left\{\xi_{m}\right\}$ выполняется соотношение $\Phi^{M}\left(\xi_{m}\right)=\Phi(O A B C)$.

ДоКАЗАТЕЛЬСтво. Зафиксируем некоторое $m$ и рассмотрим разбиение $\left\{\xi_{m}\right\}$ множества $\Phi(O A B C)$. В силу леммы 2 для произвольного $\xi_{m} \in\left\{\xi_{m}\right\}$ существует $k=k\left(\xi_{m}\right)$ такое, что $(0,0) \in \operatorname{Int}\left(\Phi^{k}\left(\xi_{m}\right)\right)$. Но $\Phi:(0,0) \rightarrow(1,1)$ и $\widetilde{K}_{\Phi} \rightarrow \Phi\left(\widetilde{K}_{\Phi}\right)$, а потому найдется $\gamma>0$ такое, что множество $\Delta:=U_{\gamma}((1,1)) \cap O A B C$, где $U_{\gamma}((1,1))$ - $\gamma$-окрестность точки $(1,1)$ в $\mathbb{R}^{2}$, принадлежит $\Phi^{k+1}\left(\xi_{m}\right)$. Посмотрим, как с ростом $j$ изменяются размеры $\Phi^{j}(\Delta)$. Обозначим $L=O B \cap \Delta$, и пусть $s(L)$-длина $L$. Покажем, что сушествует натуральное $J$ такое, что $\Phi^{J}(L)=$ $B \Phi(B)$.

Очевидно, $\Phi^{j}(L) \subset B E$ для любого $j=1,2,3, \ldots$ При этом на каждом шаге возникает следующая альтернатива:

(a) $(0,0) \cap \operatorname{Int}\left(\Phi^{j-1}(L)\right)=\varnothing$, либо

(б) $(0,0) \cap \operatorname{Int}\left(\Phi^{j-1}(L)\right) \neq \varnothing$ и точка $(0,0)$ делит $\Phi^{j-1}(L)$ на две части.

Если имеет место (а), то $s\left(\Phi^{j}(L)\right)=a s\left(\Phi^{j-1}(L)\right)$, т.е. при каждой итерации длина отрезка увеличивается в $a$ раз. Рассмотрим (б). Пусть $\tilde{L}$ - большая (любая, если они одинаковые) часть отрезка $\Phi^{j-1}(L)$. Рассмотрим $\Phi(\tilde{L})$. Так как $\Phi(0)=B$, то $B$ является одним из конщов отрезка $\Phi(\tilde{L})$. Если $(0,0) \in \Phi(\tilde{L})$, то $\Phi^{2}(\tilde{L})=B \Phi(B)$. Предположим обратное, т.е. что $(0,0) \cap \Phi(\tilde{L})=\varnothing$ и для $\Phi(\tilde{L})$ вьполняется (а). Тогда

$$
s\left(\Phi^{2}(\tilde{L})\right)=\operatorname{as}(\Phi(\tilde{L}))=a^{2} s(L)>\frac{1}{2} a^{2} s\left(\Phi^{j-1}(L)\right) .
$$

При этом одним из концов отрезка $\Phi^{2}(\tilde{L})$ является точка $\Phi(B)$. Допустим, что $\Phi(B)$ лежит левее и ниже точек $\Phi^{-1}(0)$, т.е. $\Phi^{2}(B) \in O \Phi(B)$. Это действительно так, когда $a$ такие, что $1-a \leqslant-\frac{1}{a}$ и, в частности, при $a \geqslant \frac{1+\sqrt{5}}{2}$. Тогда если $(0,0) \in$ 
$\Phi^{2}(\tilde{L})$, то $\Phi^{3}(\tilde{L})=O B$ и $\Phi^{4}(\tilde{L})=B \Phi(B)$. Если же все-таки $(0,0) \cap \Phi^{2}(\tilde{L})=\varnothing$, то для $\Phi^{2}(\tilde{L})$ вьполняется (а) и $s\left(\Phi^{3}(\tilde{L})\right)=a^{3} s(\tilde{L})>0,5 a^{3} s\left(\Phi^{j-1}(L)\right)$.

Если $a^{3}>2$, то $s\left(\Phi^{3}(\tilde{L})\right)>2 s(\tilde{L})$, а $s\left(\Phi^{j+2}(L)\right)>2 s\left(\Phi^{j-1}(L)\right)$. Как следствие получается, что если для $\Phi^{j+2}(L)$ выполняется условие (б) и $\hat{L}-$ большая часть его, то $s(\hat{L})>0,5 a^{3} s(\tilde{L})>s(\tilde{L})$ при $a>\sqrt[3]{2}$. А поскольку $\frac{1+\sqrt{5}}{2}>\sqrt[3]{2}$, то каждая итерация отображения $\Phi^{3}$ либо увеличивает длины отрезков не менее, чем в $0,5\left(\frac{1+\sqrt{5}}{2}\right)^{3}$ раз, либо же порождает отрезок, который накрывает $B \Phi(B)$. Если выбрать $i$ так, что $0,5^{i}\left(\frac{1+\sqrt{5}}{2}\right)^{3 i} s(\tilde{L})>s(B \Phi(B))$, то тогда отрезок $\Phi^{3 i}(\tilde{L})$, очевидно, накрывает отрезок $B \Phi(B)$. Полагая $J=3 i+j$, получаем, $\Phi^{J}(L)=B \Phi(B)$.

Из симметрии $\Phi^{J}(\Delta)$ относительно $B \Phi(B)$ вытекает существование $\omega>0$ такого, что $\Phi(O A B C) \cap U_{\omega}(B \Phi(B)) \subset \Phi^{J}(\Delta) \quad\left(U_{\omega}(B \Phi(B))-\omega\right.$-окрестность отрезка $B \Phi(B)$ в $\left.\mathbb{R}^{2}\right)$. Покажем, что существует натуральное $M$ такое, что $\Phi^{M}\left(\Phi(O A B C) \cap U_{\omega}(B \Phi(B))\right) \supset \Phi(O A B C) . \quad$ Рассмотрим прообразы $\Phi_{(1)}^{-p}(O A B C)$. Так как $\Phi_{(1)}^{-p}((0,0)) \in \operatorname{Int}(O B)$ и $\Phi_{(1)}^{-1}-$ сжатие, то существует точка $S \in \operatorname{Int}(O B)$, к которой при $p \rightarrow \infty$ стягиваются множества $\Phi_{(1)}^{-p}(O A B C)$. А потому найдется натуральное $P$ такое, что $\Phi_{(1)}^{-P}(O A B C) \subset U_{\omega}(B \Phi(B))$. Отсюда вытекает, что $\Phi^{P}\left(U_{\omega}(B \Phi(B))\right) \supset O A B C$ и $\Phi^{P+1}\left(U_{\omega}(B \Phi(B))\right) \supset \Phi(O A B C)$. Полагая $M=J+P+1$, имеем $\Phi^{M}\left(\xi_{m}\right)=\Phi(O A B C)$.

ЗАмЕчАниЕ. Обратим внимание на то, что при доказательстве леммы 3 мы попутно доказали следуюшее утверждение: если $a \in\left(\frac{1+\sqrt{5}}{2}, 2\right)$, то $f_{a}$ - топологически перемешивающее отображение на $\left[f_{a}^{2}(0), f_{a}(0)\right]$.

ДОКАЗАТЕЛЬСТВО ТЕОРЕМЫ. Ввиду $\Phi(B D E F)=\Phi(O A B C)$ из лемМЫ 1 вытекает, что существует окрестность $U$ такая, что $\Phi(\mathrm{Cl} U) \subset \operatorname{Int}(U)$ и $\bigcap_{j=0}^{\infty} \Phi^{j}(U)=$ $\Phi(O A B C)$. Пусть $\left\{V_{i}\right\}_{i=1}^{N}$ - произвольное покрытие $\Phi(O A B C)$ открытыми в $\mathbb{R}^{2}$ дисками и $r_{i}-$ диаметр $i$-го диска. Обозначим $r=\min \left\{r_{i}\right\}$ и выберем натуральное $m=m(r)$ так, чтобы $\operatorname{diam}\left(\xi_{m}\right)<\frac{r}{10}$. Согласно лемме 3 существует $M=M(m)$ такое, что $\Phi^{M}\left(\xi_{m}\right)=\Phi(O A B C)$. Следовательно, $\Phi^{M}\left(V_{i}\right)=\Phi(O A B C)$ для каждого $i$. Последнее означает, что $\Phi^{p}\left(V_{i}\right) \cap V_{j} \neq \varnothing$ для любых $i, j \in\{1, \ldots, N\}$ и $p \geqslant M$.

\section{Список литературы}

1. Pikovsky A.S., Grassberger P. Symmetry breaking bifurcation for coupled chaotic attractors // J. Phys. A. Math. Gen. 1991. V. 24. P. 4587-4597.

2. Losson J., Mackey $M$. Coupling induced statistical cycling in two diffusively coupled maps // Physica D. 1994. V. 72. P. 324-342.

3. Добрынский B.A. Критические множества и унимодальные отображения квадрата // Докл. РАН. 1995. Т. 341. № 4. С. 442-445.

4. Добрынский В.А. Критические множества и унимодальные отображения квадрата // Матем. заметки. 1995. Т. 58. №5. С. 669-680. 